\title{
IrRECIST Progressive Disease
}

National Cancer Institute

\section{Source}

National Cancer Institute. irRECIST Progressive Disease. NCI Thesaurus. Code C140319.

A minimum 20\% increase and minimum $5 \mathrm{~mm}$ absolute increase in total measured tumor burden compared to nadir, or irPD for non-target or new non-measurable lesions.

Confirmation of progression is recommended minimum 4 weeks after the first irPD assessment. 